\section{Análise da profilaxia da raiva humana em Campo Grande, Mato Grosso do Sul, Brasil, em 2002}

\author{
Human rabies prophylaxis in Campo Grande, \\ Mato Grosso do Sul State, Brazil, 2002
}

\section{Abstract}

Timely application of adequate treatment, speedy and thorough observation of animals, and streamlined information transfer provide quality rabies epidemiological surveillance in cases of attacks by animals. This study aims to evaluate rabies surveillance and the use of a computerized system in the surveillance process. A total of 4,168 attacks by animals were analyzed. The injuries were considered severe in $65.9 \%$ of cas es. Dogs accounted for $87.6 \%$ of the severe cases, cats $10.5 \%$, and bats $0.6 \%$. Two treatment recording sites were analyzed. According to the rabies treatment record sheet, $41.9 \%$ of the treatments were adequate; in cases with negative observation, $7.4 \%$ showed adequate treatment. According to the vaccination program, $42.2 \%$ of the prescribed treatments were correct; in cases with negative observation, $3.2 \%$ received adequate treatment. The study concluded that there should be improved prescribing and recording of treatment, as well as a search for missing cases, in order to improve the quality of the services and avoid more severe outcomes from animal attacks.

Rabies; Epidemiologic Surveillance; Prevention \& Control
Leonardo Rigo 1

Michael Robin Honer 2

\section{Introdução}

A prevenção da raiva humana é baseada no tratamento profilático anti-rábico quando houver suspeita de exposição ao vírus. Toda vez que ocorrer uma agressão por animal deve ser feita uma anamnese completa, utilizando-se a Ficha de Atendimento Anti-Rábico Humano, para ter todas as informações sobre o caso e indicar corretamente o tratamento profilático. A classificação do acidente/agressão, leve ou grave, vai depender das características do ferimento, tais como: local do corpo onde ocorreu a agressão, profundidade, extensão e número de lesões 1 .

Portanto, o tratamento profilático é baseado nas características da lesão provocada pelo animal agressor e na possibilidade de observação deste 1,2. Devem ser levadas em consideração as características do animal agressor: o estado de saúde no momento da agressão, a possibilidade de observação por dez dias, a procedência e hábitos de vida do animal. Essas observações são aplicadas apenas a agressões por cães e gatos.

Em 1995, na América Latina, 794.356 pessoas foram expostas ao risco da raiva devido a agressões de animais; em 1999, esse número cresceu para 933.260. Quanto ao número de pessoas que receberam algum tipo de tratamento pós-exposição, este subiu de 292.233 em 1995 para 310.734 em 1999. A participação de brasileiros no número de pessoas expostas ao risco 
nesse período foi de $44,5 \%$; já quanto ao percentual de pessoas que receberam tratamento pós-exposição, foi de $72,0 \% 1$.

Segundo os dados do Centro de Controle de Zoonoses de Campo Grande, Mato Grosso do Sul, Brasil, nos últimos dez anos a taxa média de agressão humana por animais foi de 6,5 / 1.000 habitantes, índice muito acima dos encontrados em estudos realizados em Curitiba (Paraná) 3, Osasco (São Paulo) 4 e Maringá (Paraná) 5 , Brasil.

No Município de Campo Grande não é registrado caso de raiva humana desde 1968, e o último caso em canino foi em 1988. Porém, em 1994 houve dois casos de raiva, diagnosticados em sagüis provenientes do Estado do Ceará, Brasil. No ano de 2001, foram diagnosticados os primeiros casos de morcegos não hematófagos positivos para raiva na região urbana do município. Naquele ano foram seis casos, e, desde então, anualmente vêm sendo diagnosticados novos casos em morcegos.

Em Campo Grande, o atendimento anti-rábico é realizado em nove Centros Regionais de Saúde. Em meados de 2001, a Ficha de Atendimento Anti-Rábico Humano foi informatizada. Ao se atender um paciente vítima de agressão, na unidade de saúde, é aberta a ficha de atendimento anti-rábico disponível em meio eletrônico, a qual fica disponível na rede interligada de computadores do sistema de Informações em Saúde da Secretaria Municipal de Saúde. A ficha pode ser acessada a qualquer momento, em qualquer terminal do sistema, permitindo a inclusão de dados referentes ao caso (tratamento, observação do animal). Ao mesmo tempo em que é feita a ficha, é aberto o sistema que registra as aplicações de vacina (Programa de Vacinação), também em rede, no qual são registradas as doses e datas de aplicação das vacinas. A informatização do Programa de Vacinação serve para o controle de imunobiológicos aplicados, bem como dos faltosos ao tratamento.

Através desse sistema são recolhidas as informações para investigação do caso e visita ao animal agressor. Como retorno, são anotados os resultados da visita, assim como outras informações relevantes à condução adequada do caso.

A informatização desses dois setores proporcionou um grande salto de qualidade à vigilância epidemiológica da raiva em Campo Grande. Ganhou-se com a guarda de informações, o fácil acesso e a agilidade na transferência de informações da observação do animal e das intercorrências relevantes de cada caso.

O presente trabalho teve como objetivo avaliar a condução da profilaxia da raiva humana em Campo Grande, em 2002, em pacientes vítimas de agressão por animal e o uso da informática no processo.

\section{Metodologia}

Para as análises do estudo, foram utilizadas as informações de 4.168 atendimentos, registrados no Serviço de Controle de Raiva e Outras Zoonoses (SECROZ). Foram descartados 117 registros duplicados, usando-se apenas um deles, e os 384 registros de agressões não investigadas, por não haver informações sobre a agressão e a condução dos casos.

Foram analisados tipo, local, extensão e profundidade da lesão; tipo de animal agressor; conduta adotada em face do caso no momento do atendimento; intervalos das visitas de observação do animal; resultados das visitas; tempo de observação do animal e motivo do encerramento desta; adequação dos tratamentos propostos e de sua condução. Na classificação de local, extensão e profundidade não foram incluídos os casos de contato indireto, embora tenham grande importância, em especial se o contato foi em região de mucosa ou em lesão prévia e extensa que viesse a expor camada mais profunda de derme.

Em caso de agressão animal a conduta recomendada é realizar a limpeza local com água e sabão, abrir uma ficha de atendimento antirábico para investigação do caso e observar o animal (cão e gato). Dependendo das características da agressão, deve-se instituir tratamento anti-rábico, o que é desnecessário em casos menos graves 6 .

Para avaliar a adequação da conduta adotada na apresentação do caso, foram considerados tipo, local, extensão e profundidade da lesão; existência comprovada de tratamento anterior e instituição ou não de tratamento vacinal.

Também foram criadas classificações para a conduta adotada, considerando-se a recomendação técnica vigente 6 : adequada - quando a conduta instituída era a forma mais adequada para evitar o risco de o paciente vir a contrair raiva, conforme as características da exposição que sofreu; inadequada - quando a conduta não garantia evitar o risco de contrair raiva; insuficiente - os casos para os quais não havia informações suficientes para formular os critérios de avaliação do tratamento; não classificada - o tratamento instituído não era compatível com as recomendações do protocolo de tratamento anti-rábico vigente.

A classificação dos tratamentos seguiu as recomendações técnicas para a profilaxia da 
raiva humana 6: tratamento 1 - três doses em dias alternados; tratamento 2 - sete doses consecutivas e dois reforços; tratamento 3 - soro anti-rábico, mais dez doses e três reforços. Foram criados alguns parâmetros para enquadrar os demais tratamentos: outros - quando o número de doses aplicadas não era compatível com os tratamentos oficiais; não definido - foram aplicadas doses de vacina, mas não havia a descrição do tratamento adotado; não tratar a conduta foi não tratar com vacina.

Para avaliar a condução do tratamento nos casos com resultado negativo da observação animal, consideraram-se, além dos critérios supracitados (para instituição do tratamento inicial), o laudo final da observação do animal, as alterações de tratamento e as doses de vacina aplicadas.

Os registros do tratamento indicado e das doses de vacina aplicadas são realizados na ficha anti-rábica e também no programa de vacinação. Embora tenham duas finalidades diferentes, atender ao Sistema Nacional de Agravos de Notificação (SINAN) e fazer o registro vacinal no prontuário do paciente, respectivamente, trata-se de uma informação que tem seu registro duplicado.

Em virtude dessa característica do registro de tratamento indicado e doses aplicadas, as análises do tratamento proposto foram feitas comparativamente entre os registros da ficha anti-rábica e do programa de vacinação.

\section{Resultados e discussão}

A identificação dos 384 (8,2\%) casos subnotificados na vigilância da raiva, e portanto não investigados como deveriam, só foi possível por causa do novo sistema informatizado, que permitiu o cruzamento de informações do atendimento ambulatorial e do registro de agressões no SECROZ.

Na análise dos 4.168 casos de agressão animal, verificou-se que o tipo de agressão que mais ocorreu foi a mordedura, $3.399(81,5 \%)$ casos, tendo como local único de agressão de maior ocorrência os membros inferiores, 1.265 $(30,4 \%)$ casos, seguido das mãos e pés, com 800 $(19,2 \%)$ casos. Essas agressões foram mais de extensão múltipla, 2.021 (48,6\%) casos; quanto à profundidade, a maioria, $2.435(58,6 \%)$ casos, foi lesão superficial.

De acordo com as descrições das lesões, estas podem ser classificadas quanto à gravidade para o risco de exposição à raiva. Na grande maioria, 2.745 (65,9\%), os casos foram considerados como lesões graves, devendo, portanto, receber algum tipo de tratamento. Ocorreram ainda $151(3,6 \%)$ casos que, em virtude da falta de informações, não puderam ser classificados.

Quanto à espécie agressora, a canina foi a maior responsável pelas agressões, com 3.651 (87,6\%) casos, seguida da felina, com 437 (10,5\%) casos. Os quirópteros ficaram em terceiro lugar, $23(0,6 \%)$ casos; outros animais domésticos, com 19 (0,5\%); animais silvestres, com 8 (0,2\%); sem informação, $28(0,7 \%)$, e rato, com 2 casos.

Para avaliar os tratamentos nas duas bases de dados pesquisadas, foi elaborada uma tabela que contemplasse os dois registros (nas fichas anti-rábicas e no programa de vacinação) de tipo de tratamento proposto para profilaxia pós-exposição à raiva, adotado no momento do atendimento ao paciente vítima de agressão animal (Tabela 1).

Foram encontradas diferenças entre os registros da ficha anti-rábica e do programa de vacinação. Os não tratados e o tratamento dois tiveram as maiores diferenças, $22,7 \%$ e $45,0 \%$, respectivamente. Considerando todos os que receberam vacina, há uma diferença de $27,8 \%$ entre os dois registros.

Entre 4.168 casos registrados, 2.177 (52,2\%) receberam doses de vacina anti-rábica. Em $1.456(66,9 \%)$ casos, o registro das doses foi realizado na ficha anti-rábica e no programa de vacinação; em 635 (29,2\%), só na ficha anti-rábica, e, em 86 (4,0\%), só no programa de vacinação. Entre os 1.456 casos com registros nos dois locais, em 387 (26,6\%), o número de doses de vacina aplicadas e registradas era maior na ficha anti-rábica; em 228 (15,7\%), o número era maior no programa de vacinação e, em 841 $(57,7 \%)$, o número de doses era igual em am-

Tabela 1

Distribuição dos registros de tratamento proposto para pós-exposição à raiva, segundo dados do Serviço de Controle da Raiva e Outras Zoonoses (SECROZ), ficha anti-rábica e programa de vacinação. Campo Grande, Mato Grosso do Sul, Brasil, 2002.

\begin{tabular}{lrrrr}
\hline Tratamento proposto & \multicolumn{2}{c}{ Ficha anti-rábica } & \multicolumn{2}{c}{$\begin{array}{c}\text { Programa de vacinação } \\
\text { n }\end{array}$} \\
& 1.110 & 26,6 & 1.082 & 26,0 \\
\hline Tratamento 1 & 126 & 3,0 & 229 & 5,5 \\
Tratamento 2 & 117 & 2,8 & 118 & 2,8 \\
Tratamento 3 & 150 & 3,6 & 113 & 2,7 \\
Outros & 2.031 & 48,7 & 2.626 & 63,0 \\
Não tratar & 634 & 15,2 & & \\
Não definido & 4.168 & 100,0 & 4.168 & 100,0 \\
Total & & & & \\
\hline
\end{tabular}


bos os registros. Entre os casos com igual número de doses aplicadas, em 717 (85,3\%), as datas de aplicação conferiam, nas demais as datas eram diferentes. Esses dados são indicativos de que o registro em dois locais abriu precedente para erros e, de certa forma, pode-se verificar que muitas vezes o tratamento não é registrado corretamente, o que seria mais uma falha na vigilância e um risco para o paciente.

Para uma pessoa que venha a ser exposta ao risco de raiva e tenha um histórico de vacinação anti-rábica anterior, o esquema vacinal de profilaxia é especial. Na ficha anti-rábica há lugar para a anotação dessa informação importante, mas em apenas $37,7 \%$ dos registros ela foi feita; os demais casos, $62,3 \%$ (2.595), não tinham o registro, conforme resultados na Tabela 2 .

Entre os 513 casos com informação de tratamento anterior, apenas $41(8,0 \%)$ tinham comprovação, ou seja, o registro das doses e da data de aplicação era compatível com a informação. O registro foi considerado duvidoso em todos os casos $(92,0 \%)$ em que não havia anotação das doses aplicadas anteriormente e/ou sua data de aplicação referia-se erroneamente ao tratamento instituído na agressão vigente.

A falta de registro de informações em ficha de investigação também foi considerada em outro trabalho realizado sobre fichas de investigação epidemiológica (Cheade MFM, et al. Comunicação pessoal; 2003).

Na Tabela 3, estão dispostos os resultados da adequação da conduta no momento do atendimento. Verificou-se que, comparando-se os locais de registros, as condutas adequadas tiveram pequena diferença $(0,6 \%)$. Já as condutas inadequadas, as com dados insuficientes para classificar e as não classificadas tiveram

Tabela 2

Distribuição dos casos, com registro de tratamento anterior e sua comprovação. Campo Grande, Mato Grosso do Sul, Brasil, 2002.

\begin{tabular}{lcc}
\hline Descrição & $\mathbf{n}$ & $\%$ \\
\hline Tratamento anterior & & \\
$\quad$ Sim & 513 & 12,3 \\
Não & 1.060 & 25,4 \\
$\quad$ Sem informação & 2.595 & 62,3 \\
& & \\
Comprovação do \\
tratamento anterior \\
$\quad$ Comprovado \\
$\quad$ Duvidoso
\end{tabular}

diferenças maiores, $31,5 \%$, 36, $1 \%$ e $24,7 \%$, respectivamente.

Para compreender melhor a dinâmica dos registros, foi realizada uma combinação entre eles, ou seja, verificou-se cada categoria de registro na ficha anti-rábica e seu correspondente no registro do programa de vacinação.

Na comparação da classificação dos registros, verificou-se que os maiores percentuais de concordâncias estão nas classificações adequada $(80,7 \%)$ e inadequada $(87,0 \%)$. Na classificação insuficiente, a concordância diminuiu $(62,2 \%)$. Esses resultados dão uma demonstração clara da confusão que há nos registros, o que pode significar que em muitos casos não houve a garantia de um tratamento adequado ao tipo de exposição sofrida (Tabela 4).

Dos 4.168 casos, 1.211 (29,0\%) foram encerrados na primeira visita; $2.221(53,3 \%)$ na segunda; 665 (16,0\%) na terceira; e $71(1,7 \%)$ na quarta. A grande maioria dos casos encerrouse com a segunda visita. Em média, as observações do animal foram encerradas em $17 \pm 19$ dias, sendo o intervalo de 11 dias o mais freqüente, mas $2.610(62,6 \%)$ casos foram encerrados com intervalo maior que 11 dias.

O ideal é que se tenha uma observação no quarto ou quinto dia após a agressão, para que se possa alterar ou suspender o tratamento proposto, conforme o resultado da observação animal. Até o quinto dia foram realizadas visitas em apenas $1.894(45,4 \%)$ casos, os quais tiveram uma observação ou pelo menos uma busca ao animal agressor nesse período.

Sete $(0,2 \%)$ casos foram encerrados antes do décimo dia, com média de sete dias, sendo o máximo de nove e o mínimo de três dias. Embora todos os animais estivessem normais na última observação, não foi respeitado o período recomendado de dez dias 1 .

Entre os 4.168 casos avaliados, $3.381(81,1 \%)$ tiveram como resultado da observação animais normais.

Houve 781 (18,7\%) casos que foram encerrados com laudo final negativo para a observação do animal, e em 307 (39,3\%) deles a falta de endereço para observação do animal agressor foi um motivo de grande importância na não observação deste. Esse valor fica acima do encontrado para os encerramentos em que a agressão fora causada por um animal de rua, contando com 237 (30,3\%) casos.

Entre os 781 casos, em virtude de o resultado final da observação ter sido negativo, alguns pacientes deveriam ter tido o seu tratamento alterado. Segundo a informação na ficha antirábica, $72,1 \%$ dos casos deveriam ter alterado o tratamento. Já de acordo com o programa de 
vacinação, são 78,9\% os casos que deveriam ter alterado o tratamento. Em $12,2 \%$ e $7,4 \%$ dos casos, respectivamente, na ficha anti-rábica e no programa de vacinação, houve dificuldade em classificar o tipo de alteração que deveria ser realizada.

Na Tabela 5, está a distribuição da adequação do tratamento instituído no momento do atendimento e como foi conduzido, após o resultado negativo da observação do animal e até o momento da coleta dos dados (mais de seis meses depois do último caso estudado).

Como resultado final dos tratamentos, ou seja, considerando o último registro sobre o tratamento instituído ao paciente cujo resultado final da observação animal foi negativo, verificou-se, nos registros da ficha anti-rábica, que somente $7,4 \%$ dos casos $(6,4 \%$ por tratamento adequado e $1,0 \%$ pela informação sobre o animal por parte da vítima) tiveram um tratamento adequado a este resultado. Já na avaliação dos registros do programa de vacinação, esse percentual foi ainda mais baixo: $3,2 \%$ dos casos $(2,2 \%$ por tratamento adequado e $1,0 \%$ pela informação sobre o animal) tiveram um tratamento adequado e seguro.

Esses dados revelam que 723 ou 756 pessoas, segundo ficha anti-rábica ou programa de vacinação, respectivamente, não tiveram um tratamento que fosse adequado para evitar o risco de contrair raiva segundo a norma de tratamento vigente. Isso ocorreu em $36,2 \%$ dos casos pelo abandono do tratamento e em $49,4 \%$ por não ter sido realizada um busca ativa e instituído um tratamento.

Estudos constataram que a não instituição de tratamento ou o seu não cumprimento eram resultados presentes em pacientes vítimas de raiva 7,8 .

Pesquisa realizada em locais de atendimento a emergências nos Estados Unidos também indicou falhas nas indicações de tratamento anti-rábico: entre os que receberam tratamento, $40,0 \%$ não deveriam ter recebido; entre os que não receberam, $6,3 \%$ deveriam ter recebido 9. Por outro lado, em estudo realizado em Curitiba 3 , os autores concluíram que 93,9\% dos atendimentos tiveram indicação correta do tratamento, e, em outro estudo na Cidade de Maringá 5, 92,0\% dos pacientes concluíram o tratamento anti-rábico prescrito.

Os resultados do presente trabalho são mais próximos dos resultados negativos das pesquisas dos Estados Unidos, pois indicam que há necessidade de o serviço buscar alternativas para um maior cumprimento das prescrições dos tratamentos para evitar o possível risco de uma pessoa contrair a doença por falhas no
Tabela 3

Distribuição da adequação da conduta adotada em face de caso apresentado como risco de exposição à raiva, segundo registros da ficha anti-rábica e programa de vacinação. Campo Grande, Mato Grosso do Sul, Brasil, 2002.

\begin{tabular}{lcccc}
\hline Adequação da conduta & \multicolumn{2}{c}{ Ficha anti-rábica } & \multicolumn{2}{c}{ Programa de vacinação } \\
& $n$ & $\%$ & $n$ & $\%$ \\
\hline Adequada & 1.745 & 41,9 & 1.757 & 42,2 \\
Inadequada & 1.028 & 24,7 & 1.500 & 36,0 \\
Insuficiente & 1.245 & 29,9 & 798 & 19,1 \\
Não classificada & 150 & 3,6 & 113 & 2,7 \\
Total & 4.168 & 100,0 & 4.168 & 100,0 \\
\hline
\end{tabular}

Tabela 4

Comparativo entre as categorias de adequação da conduta em face de risco de exposição à raiva entre os registro da ficha anti-rábica e o programa de vacinação. Campo Grande, Mato Grosso do Sul, Brasil, 2002.

\begin{tabular}{|c|c|c|c|c|}
\hline \multirow{2}{*}{$\begin{array}{l}\text { Ficha anti-rábica } \\
\text { Classificação }\end{array}$} & \multirow[b]{2}{*}{$\mathrm{n}$} & \multicolumn{3}{|c|}{ Equivalente no programa de vacinação } \\
\hline & & Classificação & $\mathrm{n}$ & \%* \\
\hline \multirow[t]{4}{*}{ Adequada } & 1.745 & Adequada & 1.409 & 80,7 \\
\hline & & Inadequada & 293 & 16,8 \\
\hline & & Insuficiente & 0 & 0,0 \\
\hline & & Não classificada & 43 & 2,5 \\
\hline \multirow[t]{4}{*}{ Inadequada } & 1.028 & Adequada & 110 & 10,7 \\
\hline & & Inadequada & 894 & 87,0 \\
\hline & & Insuficiente & 5 & 0,5 \\
\hline & & Não classificada & 19 & 1,8 \\
\hline \multirow[t]{4}{*}{ Insuficiente } & 1.245 & Adequada & 215 & 17,3 \\
\hline & & Inadequada & 212 & 17,0 \\
\hline & & Insuficiente & 774 & 62,2 \\
\hline & & Não classificada & 44 & 3,5 \\
\hline \multirow[t]{4}{*}{ Não classificada } & 150 & Adequada & 23 & 15,3 \\
\hline & & Inadequada & 101 & 67,3 \\
\hline & & Insuficiente & 19 & 12,7 \\
\hline & & Não classificada & 7 & 4,7 \\
\hline
\end{tabular}

* Percentual sobre o programa de vacinação.

tratamento. Ficou evidenciado que o sistema de vigilância apresenta falhas e há a necessidade de corrigi-las para se atingirem níveis ideais de segurança. Esses resultados também servem de alerta para que sejam tomadas providências a fim de melhorar os níveis de adequação das prescrições de tratamento.

Para que a vigilância da raiva ocorra de forma eficaz é necessária a integração entre os 
Distribuição das adequações dos tratamentos iniciados e após o resultado final da observação animal, nos casos cujo resultado da observação animal foi negativo, segundo registros da ficha anti-rábica e do programa de vacinação. Campo Grande, Mato Grosso do Sul, Brasil, 2002.

\begin{tabular}{|c|c|c|c|c|c|c|c|c|}
\hline \multirow{3}{*}{$\begin{array}{l}\text { Classificação } \\
\text { do tratamento }\end{array}$} & \multicolumn{4}{|c|}{ Tratamento iniciado } & \multicolumn{4}{|c|}{ Tratamento após resultado } \\
\hline & \multicolumn{2}{|c|}{ Ficha anti-rábica } & \multicolumn{2}{|c|}{$\begin{array}{c}\text { Programa } \\
\text { de vacinação }\end{array}$} & \multicolumn{2}{|c|}{ Ficha anti-rábica } & \multicolumn{2}{|c|}{$\begin{array}{c}\text { Programa } \\
\text { de vacinaçãa }\end{array}$} \\
\hline & $\mathrm{n}$ & $\%$ & $n$ & $\%$ & $\mathrm{n}$ & $\%$ & $\mathrm{n}$ & $\%$ \\
\hline Adequado & 261 & 33,4 & 270 & 34,6 & 50 & 6,4 & 17 & 2,2 \\
\hline Vítima informou sobre o animal & & & & & 8 & 1,0 & 8 & 1,0 \\
\hline Inadequado & 202 & 25,9 & 306 & 39,2 & 264 & 33,8 & 61 & 7,8 \\
\hline Abandonou & & & & & 231 & 29,6 & 283 & 36,2 \\
\hline Não houve tratamento & & & & & 211 & 27,0 & 386 & 49,4 \\
\hline Insuficiente & 257 & 32,9 & 141 & 18,1 & 17 & 2,2 & 26 & 3,3 \\
\hline Não classificado & 61 & 7,8 & 64 & 8,2 & & & & \\
\hline Total & 781 & 100,0 & 781 & 100,0 & 781 & 100,0 & 781 & 100,0 \\
\hline
\end{tabular}

serviços de atendimento ambulatorial e o serviço de saúde pública veterinária 10.

\section{Conclusão}

O processo de informatização possibilita cruzar informações e com isso dimensionar as subnotificações dos casos. Por meio do programa de vacinação em rede é possível verificar o acompanhamento vacinal do paciente de qualquer unidade de saúde da Rede Municipal.
Seria muito importante que fossem unificados os locais dos registros de indicação e aplicação da vacina profilática, para que se pudesse ter maior controle da aplicação da vacina anti-rábica e dos faltosos, dando qualidade ao serviço e segurança ao paciente.

Os setores envolvidos na vigilância da raiva humana devem encontrar uma solução para que, ao haver um resultado negativo na observação animal, a condução do tratamento tenha melhores resultados e qualidade, como também realizar uma busca ativa dos faltosos.

\section{Resumo}

A instituição de um tratamento adequado, a rapidez na observação do animal e no repasse de informações dão qualidade à vigilância epidemiológica em caso de agressão animal. O presente trabalho tem por objetivo avaliar a vigilância da raiva e a utilização de um sistema informatizado nesse processo. Foram avaliados 4.168 acidentes com animais. As lesões foram consideradas graves em 65,9\% das agressões. Os cães foram responsáveis por $87,6 \%$ destas, os gatos por $10,5 \%$ e os morcegos por 0,6\%. Foram analisados dois locais de registro dos tratamentos. De acordo com a ficha antirábica, 41,9\% dos tratamentos indicados estavam corretos; nos casos com resultado negativo da observação,
7,4\% tiveram um tratamento adequado. De acordo com o programa de vacinação, $42,2 \%$ dos tratamentos indicados estavam corretos; nos casos com resultado negativo da observação, 3,2\% tiveram um tratamento adequado. Concluiu-se que deve haver uma melhora na indicação e no registro dos tratamentos, bem como na busca dos faltosos, para que ocorra uma melhora na qualidade do serviço oferecido e evite-se um incidente mais grave com uma pessoa vítima de agressão animal.

Raiva; Vigilância Epidemiológica; Prevenção \& Controle 


\section{Colaboradores}

L. Rigo participou na revisão de literatura, elaboração do projeto, coleta de dados, análise dos resultados e redação do artigo final. M. R. Honer colaborou na elaboração do projeto, análise dos resultados e redação do artigo final.

\section{Referências}

1. Fundação Nacional de Saúde, Ministério da Saúde. Guia de vigilância epidemiológica. v. 2. 5a Ed. Brasília: Fundação Nacional de Saúde; 2002.

2. Instituto Pasteur. Profilaxia da raiva humana. 2a Ed. São Paulo: Instituto Pasteur; 2000. (Manual Técnico do Instituto Pasteur n.4).

3. Lange MFL, Luhn KR, Gabardo BMA. Avaliação do atendimento anti-rábico humano em Curitiba. In: Anais do V Congresso Brasileiro de Epidemiologia - EPI 2002. Rio de Janeiro: ABRASCO; 2002. p. 266.

4. Garcia RCM, Vasconcellos SA, Sakamoto SM, Lopez AC. Análise de tratamento anti-rábico humano pós-exposição em região da Grande São Paulo, Brasil. Rev Saúde Pública 1999; 33:295-301.

5. Carvalho WO, Soares DFP, Franceschi VCS. Características do atendimento prestado pelo Serviço de Profilaxia da Raiva Humana na rede municipal de saúde de Maringá-Paraná, no ano de 1997. Inf Epidemiol SUS 2002; 11:25-35.

6. Fundação Nacional de Saúde, Ministério da Saúde. Norma técnica de tratamento profilático antirábico humano. 2ạ Ed. Brasília: Fundação Nacional de Saúde; 1995.
7. Tavares-Neto J. Raiva humana: uma casuística do Estado da Bahia, Brasil. http://www.pasteur.sau de.sp.gov.br/informacoes/anais/seminario_inter nacional/resumo_6_6.htm (acessado em 20/Mar/ 2005).

8. Centro Panamericano de Fiebre Aftosa. IX REDIPRA - reunión de directores de los programas nacionales de control de rabia en América Latina informe final. Santa Cruz de la Sierra: Centro Panamericano de Fiebre Aftosa/Salud Pública Veterinaria/Organización Panamericana de la Salud/Organización Mundial de la Salud; 2003.

9. Moran GJ, Talan DA, Mower W, Newdow M, Ong $\mathrm{S}$, Nakase JY, et al. Appropriateness of rabies postexposure prophylaxis treatment for animal exposures. Emergency ID Net Study Group. JAMA 2000; 284:1001-7.

10. Nova AV, Rengeli FS, Hinrichsen SML. Raiva. In: Veronesi R, Focaccia R, organizadores. Tratado de infectologia. São Paulo: Ateneu; 1996. p. 476-87.

Recebido em 08/Mar/2005

Versão final reapresentada em 23/Mai/2005 Aprovado em 03/Jun/2005 